\title{
The natriuretic peptide/guanylyl cyclase-A system functions as a stress-responsive regulator of angiogenesis in mice
}

\author{
Michaela Kuhn, ${ }^{1}$ Katharina Völker, ${ }^{1}$ Kristine Schwarz, ${ }^{1}$ Javier Carbajo-Lozoya, ${ }^{2}$ Ulrich Flögel, ${ }^{3}$ \\ Christoph Jacoby, ${ }^{3}$ Jörg Stypmann, ${ }^{4}$ Martin van Eickels, ${ }^{5}$ Stepan Gambaryan, 6,7 \\ Michael Hartmann, ${ }^{1}$ Matthias Werner, ${ }^{1}$ Thomas Wieland,2 Jürgen Schrader, ${ }^{3}$ and Hideo A. Baba ${ }^{8}$

\begin{abstract}
${ }^{1}$ Institute of Physiology, University of Würzburg, Würzburg, Germany. ${ }^{2}$ Institute of Experimental and Clinical Pharmacology and Toxicology, University of Heidelberg, Mannheim, Germany. ${ }^{3}$ Department of Cardiovascular Physiology, Heinrich-Heine-University, Düsseldorf, Germany. ${ }^{4}$ Department of Cardiology and Angiology, University Hospital Münster, Münster, Germany. ${ }^{5}$ Institute of Physiology II, Rheinische Friedrich-Wilhelms-University, Bonn, Germany. ${ }^{6}$ Institute of Clinical Biochemistry and Institute of Pathobiochemistry, University of Würzburg, Würzburg, Germany. ${ }^{7}$ Sechenov Institute of Evolutionary Physiology and Biochemistry of the Russian Academy of Sciences, St. Petersburg, Russia. ${ }^{8}$ Institute of Pathology and Neuropathology, University of Duisburg-Essen, Essen, Germany.
\end{abstract}

\begin{abstract}
Cardiac atrial natriuretic peptide (ANP) and B-type natriuretic peptide (BNP) modulate blood pressure and volume by activation of the receptor guanylyl cyclase-A (GC-A) and subsequent intracellular cGMP formation. Here we report what we believe to be a novel function of these peptides as paracrine regulators of vascular regeneration. In mice with systemic deletion of the GC-A gene, vascular regeneration in response to critical hind limb ischemia was severely impaired. Similar attenuation of ischemic angiogenesis was observed in mice with conditional, endothelial cell-restricted GC-A deletion (here termed EC GC-A KO mice). In contrast, smooth muscle cell-restricted GC-A ablation did not affect ischemic neovascularization. Immunohistochemistry and RT-PCR revealed BNP expression in activated satellite cells within the ischemic muscle, suggesting that local BNP elicits protective endothelial effects. Since within the heart, BNP is mainly induced in cardiomyocytes by mechanical load, we investigated whether the natriuretic peptide/GC-A system also regulates angiogenesis accompanying load-induced cardiac hypertrophy. EC GC-A KO hearts showed diminished angiogenesis, mild fibrosis, and diastolic dysfunction. In vitro BNP/GC-A stimulated proliferation and migration of cultured microvascular endothelia by activating cGMP-dependent protein kinase I and phosphorylating vasodilatorstimulated phosphoprotein and p38 MAPK. We therefore conclude that BNP, produced by activated satellite cells within ischemic skeletal muscle or by cardiomyocytes in response to pressure load, regulates the regeneration of neighboring endothelia via GC-A. This paracrine communication might be critically involved in coordinating muscle regeneration/hypertrophy and angiogenesis.
\end{abstract}

\section{Introduction}

Atrial and B-type natriuretic peptides (ANP and BNP) activate a common guanylyl cyclase-A (GC-A) receptor expressed in many tissues, thereby increasing intracellular cGMP (1). The important endocrine role of cardiac ANP in blood pressure/volume homeostasis has been emphasized by the phenotype of genetic mouse models. Targeted deletion of the peptide or its receptor leads to severe, chronic arterial hypertension, hypervolemia, and cardiac hypertrophy (2-4). The physiological role of BNP is less clear. Intriguingly, although BNP and ANP signal through the same receptor, mice without $B N P$ exhibit a phenotype different from that of ANP-deficient mice. Whereas BNP-deficient mice do not have hypertension or cardiac hypertrophy, they are susceptible to cardiac fibrosis (5). Under physiological conditions (in the absence

Conflict of interest: The authors have declared that no conflict of interest exists. Nonstandard abbreviations used: ANP, atrial natriuretic peptide; BNP, B-type natriuretic peptide; EC GC-A KO, endothelium-restricted GC-A deletion; GC-A, guanylyl cyclase-A; GC-A KO, GC-A gene knockout; LDPI, laser Doppler perfusion imaging; MLEC, microvascular lung endothelial cell; MRA, magnetic resonance angiography; $\mathrm{NP}$, natriuretic peptide; RFPEC, rat epididymal fat pad endothelial cell; SMC GC-A $\mathrm{KO}$, smooth muscle-restricted GC-A deletion; TAC, transverse aortic constriction; VASP, vasodilator-stimulated phosphoprotein.

Citation for this article: J. Clin. Invest. 119:2019-2030 (2009). doi:10.1172/JCI37430 of cardiac pressure or volume overload), the peripheral circulating plasma concentrations of BNP are much lower than those of ANP (6). Also, the affinity of BNP for binding to GC-A is nearly 10-fold lower than that of ANP (6). Thus, it is possible that BNP mainly acts as a local paracrine factor modulating cellular proliferation and tissue remodeling within the heart (where it is constitutively expressed in ventricular myocytes) as well as in other tissues (where it is expressed, for instance, in response to hypoxia) $(7,8)$.

In particular, several studies suggested that the natriuretic peptide (NP)/GC-A system could be involved in the stimulation of postischemic vascular regeneration. The GC-A receptor is densely expressed in endothelia (1), and low concentrations of ANP stimulated proliferation and migration of cultured human macrovascular endothelial cells (9). An increase in circulating BNP levels resulting from targeted overexpression of the BNP gene in the liver accelerated vascular regeneration in limb ischemia experimentally generated in mice by femoral artery ligation (10). Moreover, a recent clinical study showed the therapeutic potential of intravenously administered recombinant human ANP (carperitide) in patients with peripheral arterial diseases (11). Taken together, these studies demonstrate the protective pharmacological effects of high ANP or BNP concentrations. However, they do not unravel whether the endogenous NP/GC- 
A

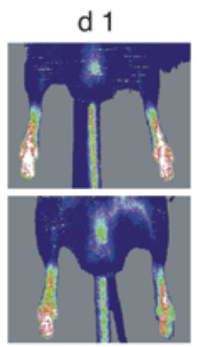

d 14

d 1
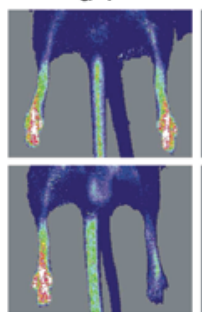

d 14

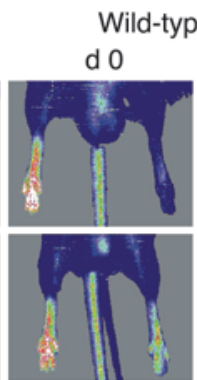

d 21

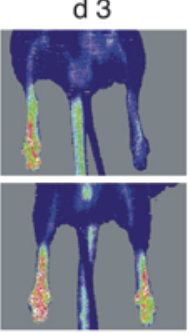

d 28

d 0

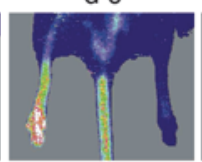

d 3

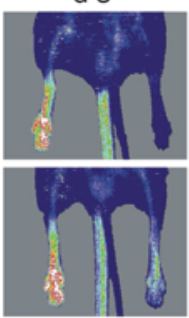

d 28

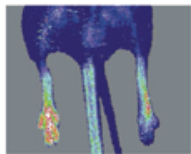

d 21

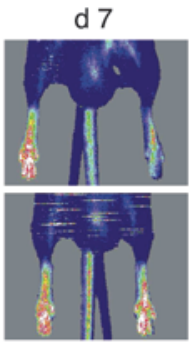

d 35

d 7

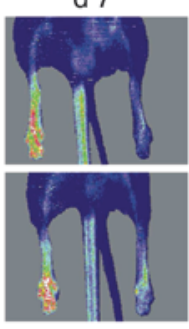

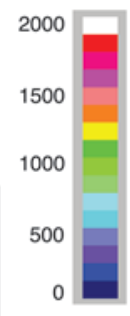

d 35
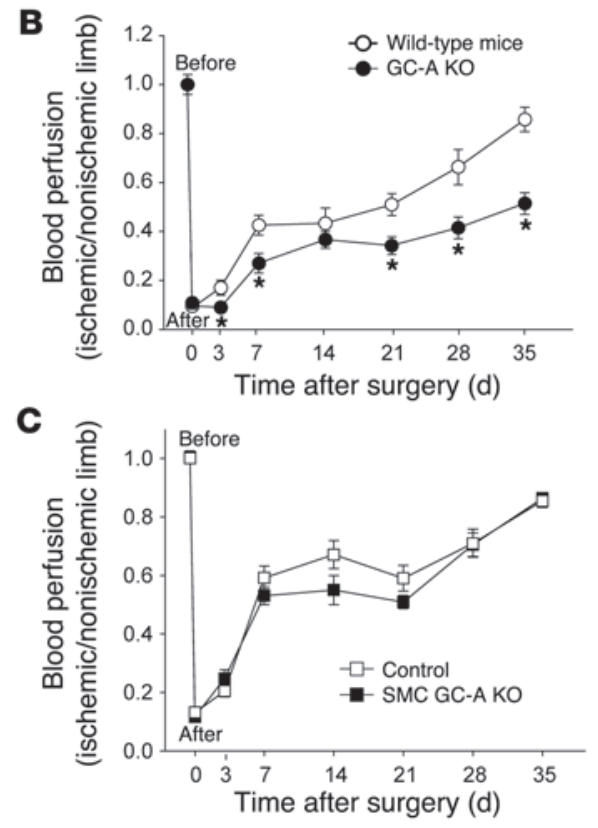

D

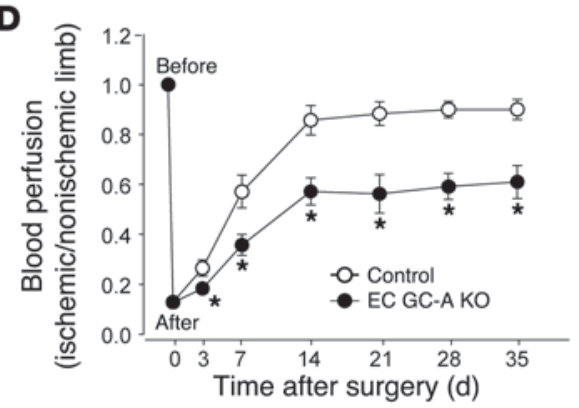

Figure 1

Inhibition of the NP/GC-A system impairs blood flow recovery after hind limb ischemia. Control mice (with normal GC-A expression levels) and mice with global (GC-A KO) (A and B), smooth muscle-restricted (SMC GC-A KO) (C), or endothelium-restricted deletion of GC-A (EC GC-A KO) (D) underwent left femoral artery excision. Hind limb blood flow was assessed by LDPI immediately before and after ligation (day 0 ) and on postoperative days $3,7,14,21,28$, and 35 . Typical LDP images of global GC-A KO and respective wild-type mice are shown in A. Perfusion in the ischemic limb is expressed relative to that in the nonischemic limb in B-D. ${ }^{*} P<0.05$ versus respective wild-type or control mice (10-12 mice per genotype). Two-way ANOVA results showed a significant genotype-treatment interaction; $P<0.05$.

A system modulates ischemic vascular regeneration. The pathophysiology of reperfusion after critical ischemia is complex. It involves angiogenesis and arteriogenesis, conditions that result from the interaction of various cell types, including inflammatory cells, platelets, and vascular cells such as pericytes, endothelia, smooth muscle cells, as well as fibroblasts (12). With the exception of platelets and leukocytes, all these cell types express the GC-A receptor (1). The present study was designed to evaluate (a) whether endogenous NPs participate in the induction of postischemic vascular regeneration; (b) the vascular cell type(s) mediating their potential protective effects; and (c) the role of NPs in coronary angiogenesis accompanying load-responsive cardiac hypertrophy. We studied functional recovery and angiogenesis after critical hind limb ischemia, a model of peripheral arterial disease, and after cardiac pressure overload. To dissect the role of the endogenous NP/GC-A system, we took advantage of genetically engineered mice with global (GC-A KO; ref. 3) deletion or cell-specific endothelial (EC GC-A KO) or smooth muscle-restricted deletion of GC-A (SMC GC-A KO) $(13,14)$.
Our findings indicate that endogenous NPs function as stressresponsive regulators of angiogenesis in the mouse hypertrophic heart and ischemic hind limb.

\section{Results}

Reperfusion after hind limb ischemia was impaired in mice with global or endothelial deletion of GC-A. First we studied vascular regeneration after limb ischemia experimentally generated in mice by femoral artery excision. Serial blood flow measurements by laser Doppler perfusion imaging (LDPI) revealed that recovery of limb perfusion was severely delayed and impaired in GC-A KO mice (3) compared with the respective wild-type mice (Figure 1, A and B). The ratio of perfusion in ischemic relative to that in nonischemic hind limb was $0.27 \pm 0.04$ for GC-A KO versus $0.42 \pm 0.04$ for wild-type mice at day $7(P=0.02)$ and $0.51 \pm 0.04$ versus $0.85 \pm 0.05$ at day 35 $(P=0.0001)$, respectively. Thus, whereas in wild-type mice, perfusion of the ischemic limb was nearly restored at 5 weeks of occlusion, in GC-A KO mice, limb perfusion remained significantly impaired during the whole 5 -week observation period. 

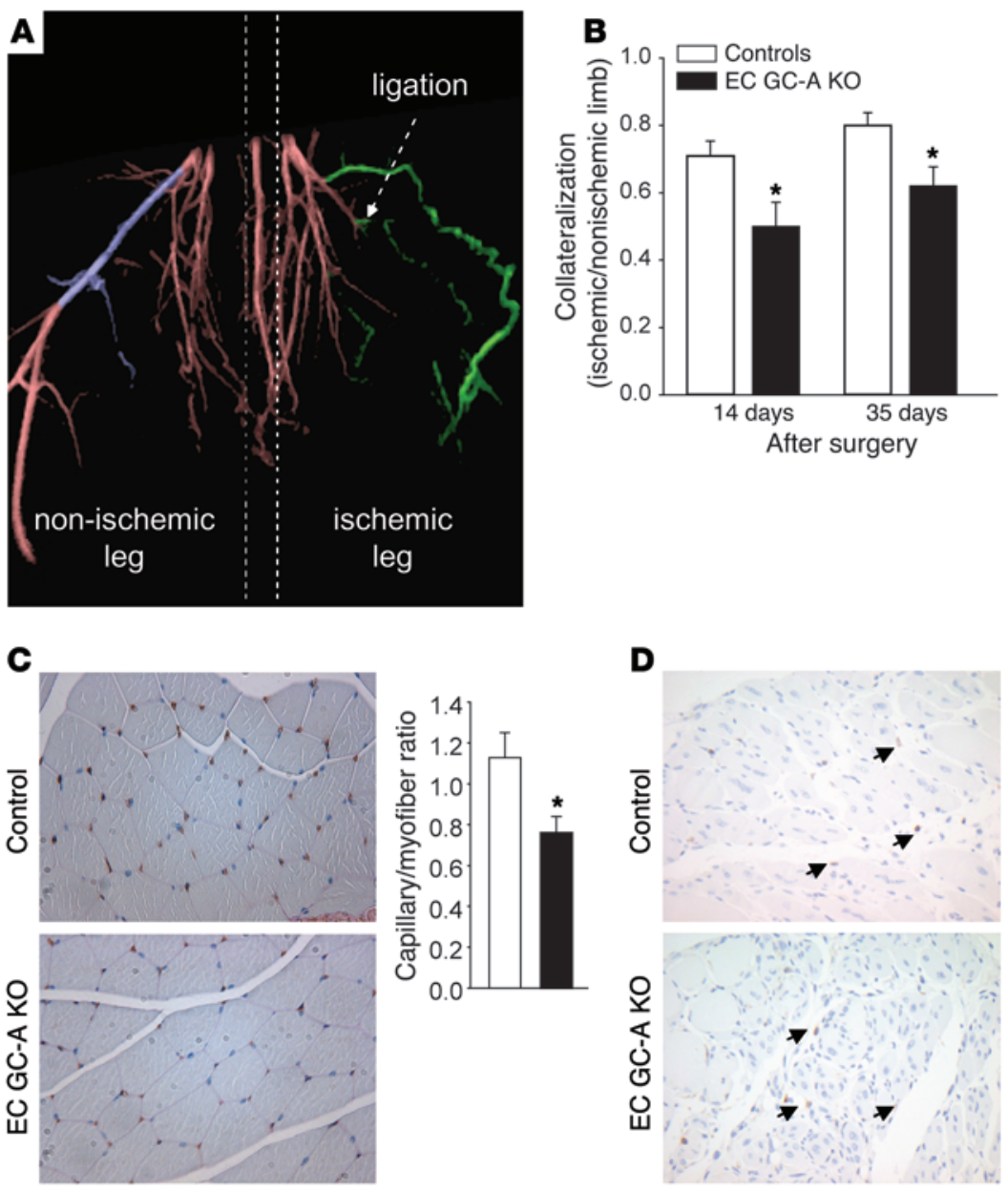

Figure 2

Ischemia-induced formation of collateral vessels and angiogenesis were impaired in EC GC-A KO mice. (A) MRA depiction of collateral vessel formation (green) 2 weeks after hind limb ischemia (front view). The reference vessel segment on the contralateral side is colored blue. (B) Quantification of MRA images 14 and 35 days after ischemia demonstrated that collateral vessel formation and replenishment of flow in the distal vessels were significantly impaired in EC GC-A KO mice. Collateralization in the ischemic limb is expressed relative to that in the nonischemic limb. ${ }^{*} P<0.05$ versus respective control mice (12 mice per each genotype). Two-way ANOVA results showed a significant genotype-treatment interaction; $P<0.05$. (C and $\mathbf{D})$ Gastrocnemius muscle tissue was harvested 7 days after ischemia. Sections were stained with the endothelial marker isolectin (brown) and PAS for quantification of capillary density and myocytes (C) and with anti-CD45 antibody (brown) for analysis of inflammation within areas of muscle regeneration (D). Representative muscle sections demonstrate fewer capillaries but similar numbers of infiltrating CD45-positive leukocytes (arrows in D) in the ischemic hind limb of EC GC-A KO mice as compared with respective control littermates. Data are summarized in the bar graphs. ${ }^{*} P<0.05$ versus control mice (5 mice per genotype). Original magnification of all histological figures, $\times 400$.

To determine which vascular cell type was mediating the protective effects of endogenous ANP and/or BNP in postischemic reperfusion, we extended our studies to mice with cell-restricted endothelial (GC-A flox/flox $\times$ Tie2Cre: EC GC-A KO) or smooth muscle GC-A deletion (GC-A flox/flox $\times$ SM22Cre: SMC GC-A KO) $(13,14)$. As shown in Figure 1C, at all evaluation times, the extent of restoration of perfusion in the ischemic hind limb was similar in SMC

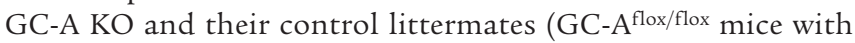
normal GC-A expression levels). In contrast, postischemic reperfusion was severely delayed and impaired in EC GC-A KO mice (Figure 1D). Fourteen days after femoral artery ligation, blood

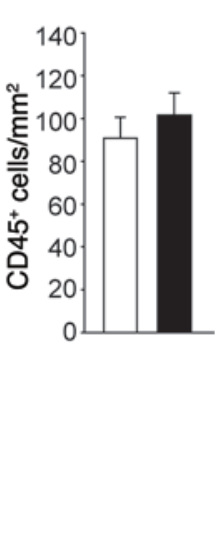

flow in the ischemic hind limb had returned almost to normal in control mice (Figure 1D). Conversely, blood flow recovery was markedly reduced in EC GC-A $\mathrm{KO}$ mice. Even 35 days after femoral artery occlusion, blood flow in the ischemic hind limb was only $52 \% \pm 6 \%$ of that in the nonischemic leg.

Impaired ischemic limb arteriogenesis and angiogenesis in mice with endothelial ablation of GC-A. Blood flow recovery in this animal model of chronic hind limb ischemia depends on both angiogenesis, the sprouting of capillaries, and arteriogenesis, the growth and remodeling of preexisting arterioles into physiologically relevant arteries (12). Because laser Doppler measurements were restricted to the measurement of superficial skin blood flow, we applied magnetic resonance angiography (MRA) to assess deep arteriogenesis in EC GC-A KO mice and respective control littermates 14 and 35 days after femoral artery ligation (15). Figure 2A shows the femoral vessel system of a mouse 2 weeks after ligation of the left femoral artery (arrow), demonstrating the extent of arteriogenesis (green). The femoral vessel distal to the ligation site was partly reperfused (Figure $2 \mathrm{~A})$, and at least one collateral vessel with a typical "corkscrew" appearance was clearly visible in the angiogram. To account for differences in individual animal size and vessel geometry, we normalized the number of newly developed vessels to that in an anatomically defined artery segment on the contralateral side (blue; Figure 2A) (15). Figure $2 \mathrm{~B}$ shows that the extent of collateralization in the ischemic hind limb (calculated as ratio to that in the nonischemic limb) was significantly lower in EC GC-A KO mice as compared with their control littermates: $50 \% \pm 7 \%$ vs. $71 \% \pm 4 \%$ at 14 days $(P=0.027)$ and $62 \% \pm 6 \%$ vs. $80 \% \pm 4 \%$ at 35 days after ligation $(P=0.01,12$ mice per genotype). These results corroborate the LDPI measurements.

Compatible with these results, histology and isolectin stainings demonstrated that capillary density in the gastrocnemius muscle of the ligated leg was significantly lower in EC GC-A KO as compared with control mice (Figure 2C). No significant genotypedependent differences were seen in the number of CD45-positive 

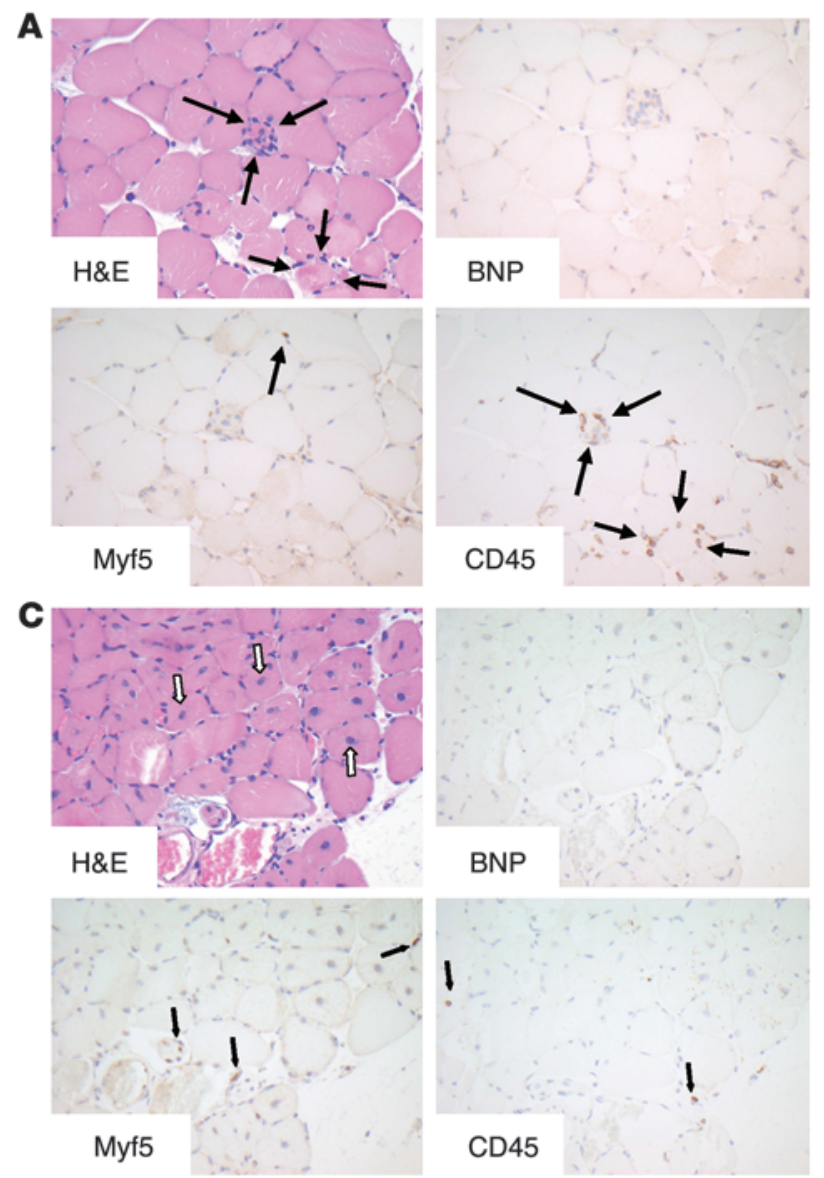
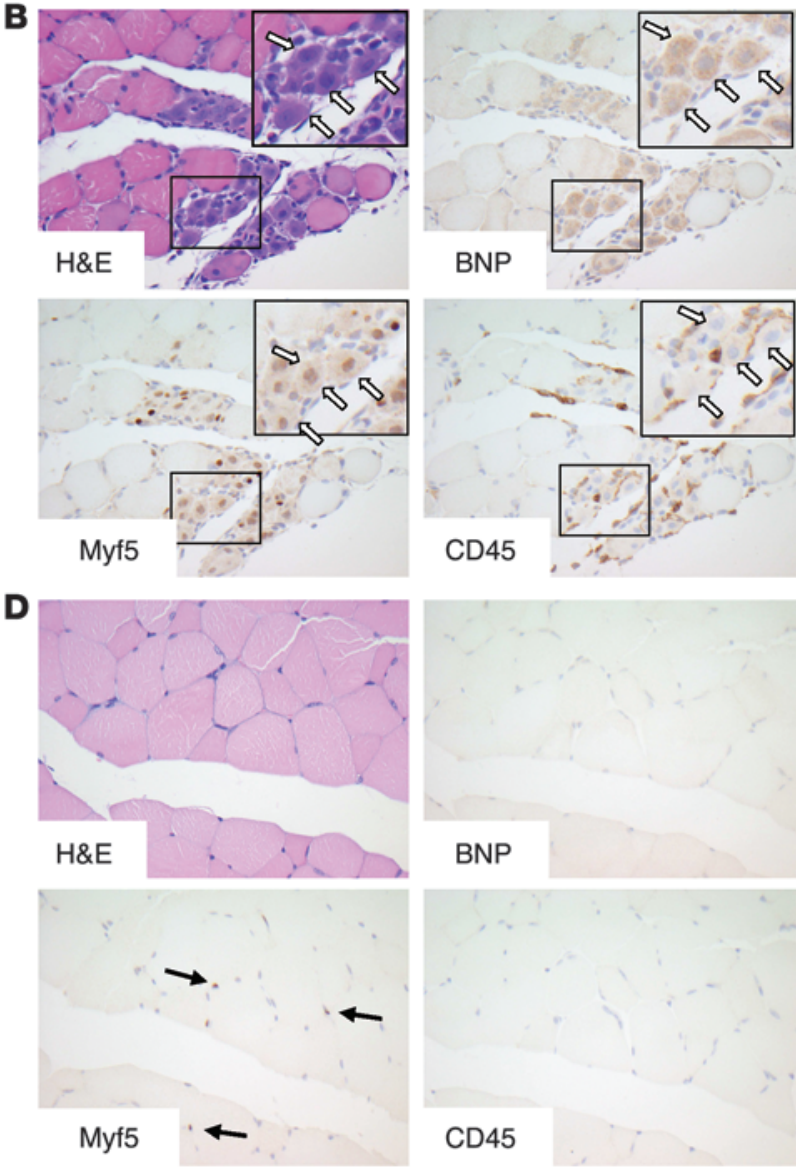
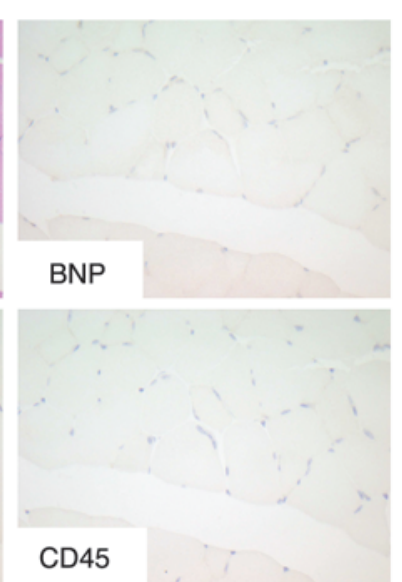

Figure 3

Immunohistochemistry on serial sections demonstrates expression of BNP in activated satellite cells of the ischemic hind limb. (A) Twenty-four hours after ischemia. Arrows on H\&E-stained sections indicate necrosis of muscle fibers, surrounded by CD45-positive leukocytes. Only a few quiescent Myf5-positive satellite cells (lower left, arrow) are detected. None of these cell types contains immunoreactive BNP. (B) Three days after ischemia. H\&E staining shows activated satellite cells with enlarged vesicular nuclei and basophilic cytoplasm (upper left, white arrows), which express Myf5 in nuclei and to a lesser extent in cytoplasm. These activated satellite cells contain immunoreactive BNP in their cytoplasm (upper right, white arrows). Clusters of satellite cells are surrounded by CD45-positive inflammatory cells but are themselves CD45-negative (lower right, white arrows). The boxed regions within the main panels denote a cluster of satellite cells at the original $(\times 400)$ and at higher magnification (×800). (C) Two weeks after ischemia. H\&E stainings demonstrate regenerating muscle fibers with centrally located nuclei (upper left, white arrows). Only a few quiescent Myf5-positive satellite cells and CD45-positive leukocytes are detected (lower panels, black arrows). No specific BNP staining is visible (upper right). (D) Nonischemic skeletal muscle of the contralateral limb shows vital muscle fibers without enhanced cellularity (H\&E). Few quiescent satellite cells with Myf5 in nuclei are detected (lower left, black arrows). Leukocytes are absent. No specific BNP staining is visible (upper right). Original magnification for all panels, $\times 400$.

leukocytes infiltrating areas of muscle regeneration (Figure 2D) or in necrosis (data not shown). Taken together, these data demonstrate that endothelial NP/GC-A signaling plays a critical role in postischemic arteriogenesis and angiogenesis.

$B N P$ is expressed in activated satellite cells within the ischemic bind limb. Immunohistochemical stainings for BNP and for markers of leukocytes (CD45; ref. 10) and satellite cells (Myf5; ref. 16) were performed using serial sections of paraffin-embedded skeletal muscle tissue obtained $1,3,7$, and 14 days after ischemia. One day after ischemia, areas of muscle fiber necrosis were surrounded by infiltrating leukocytes (Figure 3A). Some quiescent satellite cells were scattered between the muscle fibers, showing Myf5positive signals in nuclei (Figure 3A). At this early time point, no immunoreactive BNP was present. Three days after ischemia, foci of activated, proliferating satellite cells were detectable, showing vesicular nuclei and basophilic cytoplasm in H\&E staining and immunoreactive Myf5 primarily in the nucleus and to a lesser extent in cytoplasm (Figure 3B). These activated satellite cells strongly expressed immunoreactive BNP in the cytoplasm (Figure 3B). They were surrounded by some (BNP-negative) leukocytes (Figure 3B). The same observations were made in muscle sections obtained 7 days after ischemia (data not shown). Two weeks after ischemia, regenerating muscle fibers were easily recognizable by their centrally located nuclei (Figure 3C). Some quiescent satellite cells and inflammatory cells that did not express immunoreactive BNP (Figure 3C) were present. Edema or bleeding in the ischemic hind limb tissues was not observed at any time point. At all evaluation times, the contralateral nonischemic hind limb muscle contained only a few quiescent satellite cells, and these did not express BNP (Figure 3D). 


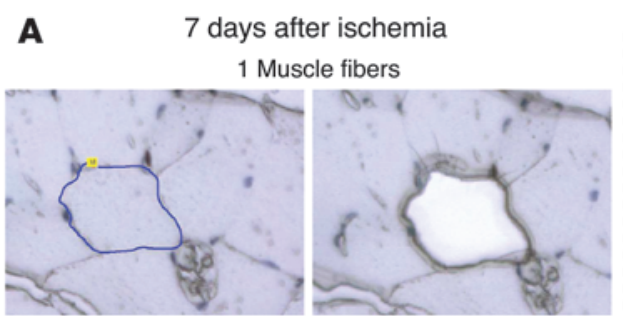

2 Satellite cells
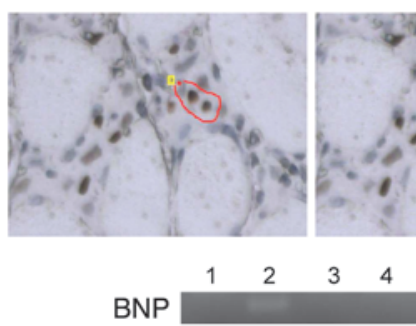

Cyclophilin A

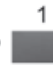

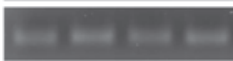
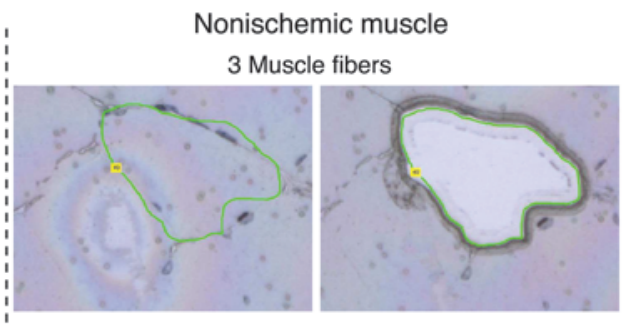

4 Satellite cells

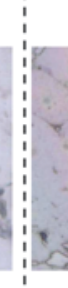

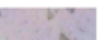
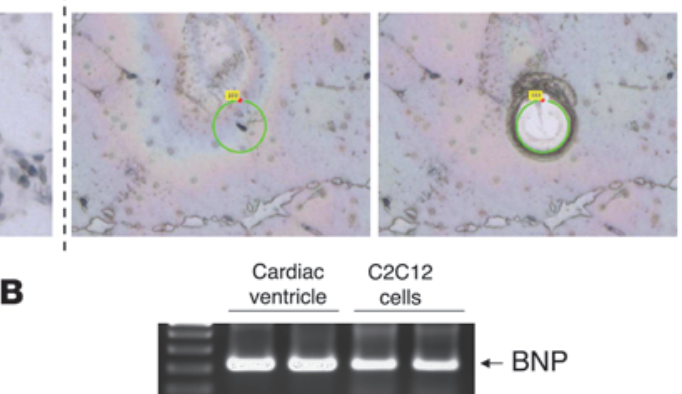

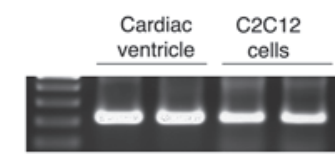

$\leftarrow$ BNP

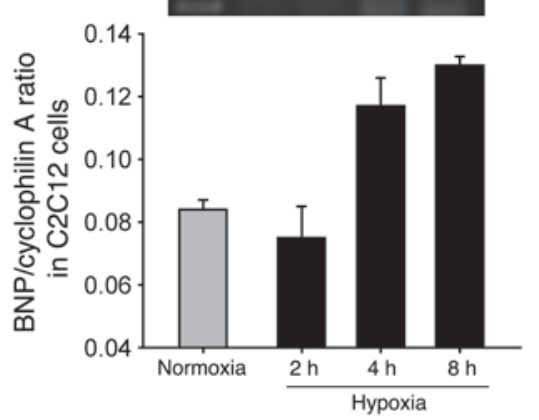

Figure 4

BNP mRNA expression in lasermicrodissected satellite cells and cultured C2C12 cells. (A) Myf5immunoreactive satellite cells and Myf5-negative muscle fibers were laser-microdissected from ischemic and nonischemic muscle. Top: Photographs showing the cells of interest before and after laser cut (original magnification, $\times 400$ ); numbers correspond to the lanes in the blot below. Bottom: By RT-PCR analyses, BNP mRNA expression was detected exclusively in satellite cells of the ischemic muscle. Cyclophilin A was used as reference gene. The signal is very weak because only very low amounts of RNA were obtained from paraffin-embedded tissue sections. (B) In C2C12 cells, BNP mRNA is strongly expressed and is induced by hypoxia.
To verify these results, we studied BNP mRNA expression in laser-microdissected satellite cells and in cultured C2C12 cells, a mouse myoblast cell line. We excised satellite cells (identified by immunostaining) and muscle fibers from the ischemic and nonischemic muscle and analyzed them for expression of BNP by RT-PCR. As shown in Figure 4A, only activated satellite cells from the ischemic muscle expressed BNP. No expression was detected in muscle fibers or in satellite cells of the nonischemic hind limb. Because only very small amounts of RNA were obtained from cells dissected from these paraffin-embedded tissue sections, the products of RT-PCR for both BNP and cyclophilin A (used as reference gene) were very weak (Figure 4A). Last, quantitative real time RT-PCR demonstrated that BNP mRNA was strongly expressed in $\mathrm{C} 2 \mathrm{C} 12$ cells and was induced by hypoxia (Figure 4B). Taken together these in vivo/in vitro data suggest that expression of BNP induced in activated satellite cells of the ischemic muscle stimulates angiogenesis via endothelial GC-A.

Endothelial GC-A ablation in mice impairs pressure overload-angmented cardiac angiogenesis. Cardiac hypertrophy and angiogenesis are coordinately regulated during physiological or adaptive cardiac growth (17). ANP and especially BNP are among the earliest and most sensitive stress-responsive fetal genes induced in cardiomyocytes in response to pressure overload. To test whether this cardiac induction of NPs participates in the regulation of coronary angiogenesis, we subjected EC GC-A KO mice and respective control littermates to transverse aortic constriction (TAC). Seven-week-old control and EC GC-A KO mice were first subjected to echocardiography, which revealed no differences in ventricular performance at this young age (Table 1). Both groups of mice were then subjected to TAC and assessed 10 days afterward. The induced pressure gradient $(\sim 50 \mathrm{mmHg})$ and the load-provoked increases in left-ventricular mass were similar in EC GC-A KO mice and controls (see Table 1). Left-ventricular systolic function was not altered, as fractional shortening and ejection fraction remained optimal (Table 1). However, in contrast to the control mice, EC GC-A KO mice developed significant left-ventricular diastolic dysfunction, indicated by the $\mathrm{E} / \mathrm{A}$ ratio of the transmitral inflow velocities, which increased in control but decreased in EC GC-A KO mice in response to TAC ( $P=0.017$ between genotypes; Table 1$)$. The E/A ratio is a commonly used index of left-ventricular diastolic function (18). Histological analyses showed that hearts of EC GC-A KO mice exhibited mild fibrosis, as assessed by picrosirius red staining (Figure $5 \mathrm{~A}$ ). Assessment of capillary and endothelial content by isolectin stainings revealed a substantial decrease in EC GC-A KO compared with control hearts (Figure 5B, top). Quantification showed significantly fewer capillaries per single cardiomyocyte (Figure 5B, bottom). In contrast, under baseline conditions (without TAC) capillary densities were not significantly different in control and EC GC-A KO hearts (data not shown). These observations indicate that endothelial GC-A activation participates in angiogenesis during the early compensatory phase of pathologic hypertrophy.

NPs stimulate proliferation, migration, and angiogenic sprouting of cultured microvascular endothelial cells. The functional responses to ANP and BNP were studied in spontaneously immortalized, well-charac- 
Table 1

Echocardiography

\begin{tabular}{lcccc} 
& \multicolumn{2}{c}{ Before TAC } & \multicolumn{2}{c}{ After TAC } \\
& Controls & EC GC-A KO & Controls & EC GC-A KO \\
& $n=14$ & $n=14$ & $n=14$ & $n=14$ \\
HR (bpm) & $476 \pm 7$ & $462 \pm 13$ & $494 \pm 4$ & $436 \pm 6$ \\
FS (\%) & $52 \pm 0.4$ & $50 \pm 2$ & $47 \pm 1.4$ & $47 \pm 1.3$ \\
EF (\%) & $78 \pm 1.1$ & $75 \pm 2.2$ & $75 \pm 1.66$ & $75 \pm 1.38$ \\
LV mass (mg) & $62 \pm 3.3$ & $68 \pm 3.4$ & $99 \pm 5.55^{A}$ & $106 \pm 5.83^{A}$ \\
CO (ml/min) & $16 \pm 0.83$ & $16 \pm 0.81$ & $22 \pm 1.11$ & $23 \pm 1.38$ \\
Cl (ml/min/g) & $0.89 \pm 0.04$ & $0.97 \pm 0.06$ & $1.06 \pm 0.04$ & $1.09 \pm 0.05$ \\
E/A ratio & $1.8 \pm 0.16$ & $1.9 \pm 0.19$ & $3.2 \pm 0.72$ & $1.3 \pm 0.448$ \\
TAC-grad (mmHg) & $0 \pm 0$ & $0 \pm 0$ & $47 \pm 3.6$ & $48 \pm 5.1$ \\
& & & & \\
\hline Analyses on
\end{tabular}

Analyses of cardiac mass and cardiac function of control and EC GC-A KO mice before and 10 days after TAC. HR, heart rate; FS, fractional shortening; EF, ejection fraction; $\mathrm{CO}$, cardiac output; $\mathrm{Cl}$, cardiac index; $\mathrm{E} / \mathrm{A}$ ratio, ratio of the $\mathrm{E}$-wave (maximum early flow velocity mitral valve) to the A-wave (maximum late flow velocity mitral valve); TAC-grad, pressure gradient induced by TAC. ${ }^{A} P<0.05$ vs. mice before TAC. ${ }^{B} P<0.05$ vs. control mice. $n=14$ mice per genotype.
Previous studies indicated that NPs modulate the release of VEGF from cultured SMCs (21). VEGFstimulated proliferation of cultured ECs, triggered by endothelial NO synthase activation (22), was also shown to require intracellular signaling through cGMP and PKG I (23). We therefore studied whether the proangiogenic effects of NPs could be indirectly mediated by enhanced secretion and autocrine/paracrine action of endogenous VEGF. In the supernatant of RFPECs cultured for 4 and 24 hours, $1.3 \pm 0.1$ and $28.2 \pm 1.2$ $\mathrm{pg} / \mathrm{ml}$ VEGF-A, respectively, were detected with a specific ELISA. ANP or BNP ( $1 \mathrm{nM}$ to $1 \mu \mathrm{M}$, incubation during 4 and 24 hours) did not change the release of VEGF-A into supernatants. Furthermore, treatment with PTK787/ZK222584 (100 nM), a potent inhibitor of VEGF receptor tyrosine kinases (24), reduced basal sprouting of RFPECs but did not alter the concentration dependence of BNP-induced sprouting (Figure 6D) and proliferation (data not shown). These results indicate that NPs can directly potentiate endothelial regeneration via stimulation of the GC-A/PKG I path-

terized microvascular endothelial cells from rat epididymal fat pad endothelial cells (RFPECs) (19). As shown in Figure 6, A and B, BNP increased intracellular cGMP content and activated cGMP-dependent protein kinase (PKG) I (demonstrated by phosphorylation of vasodilator-stimulated phosphoprotein [VASP] at Ser236, the PKG I preferred site; ref. 20). Similar effects were obtained with ANP (data not shown). BNP stimulated proliferation, migration, and angiogenic sprouting of RFPECs with a similar concentration dependence (Figure 6C). These proangiogenic responses were largely suppressed in the presence of Rp-8-pCPT-cGMPs $(10 \mu \mathrm{M})$, a specific PKG I inhibitor. The effects of ANP, but not $\mathrm{CANP}_{4-23}$, a ligand for the NP clearance receptor (NPR-C), were similar to those of BNP (Figure $6 \mathrm{D})$. Notably, the maximal NP-induced proangiogenic effects were as strong as those evoked by VEGF-A (50 ng/ml; Figure 6D).
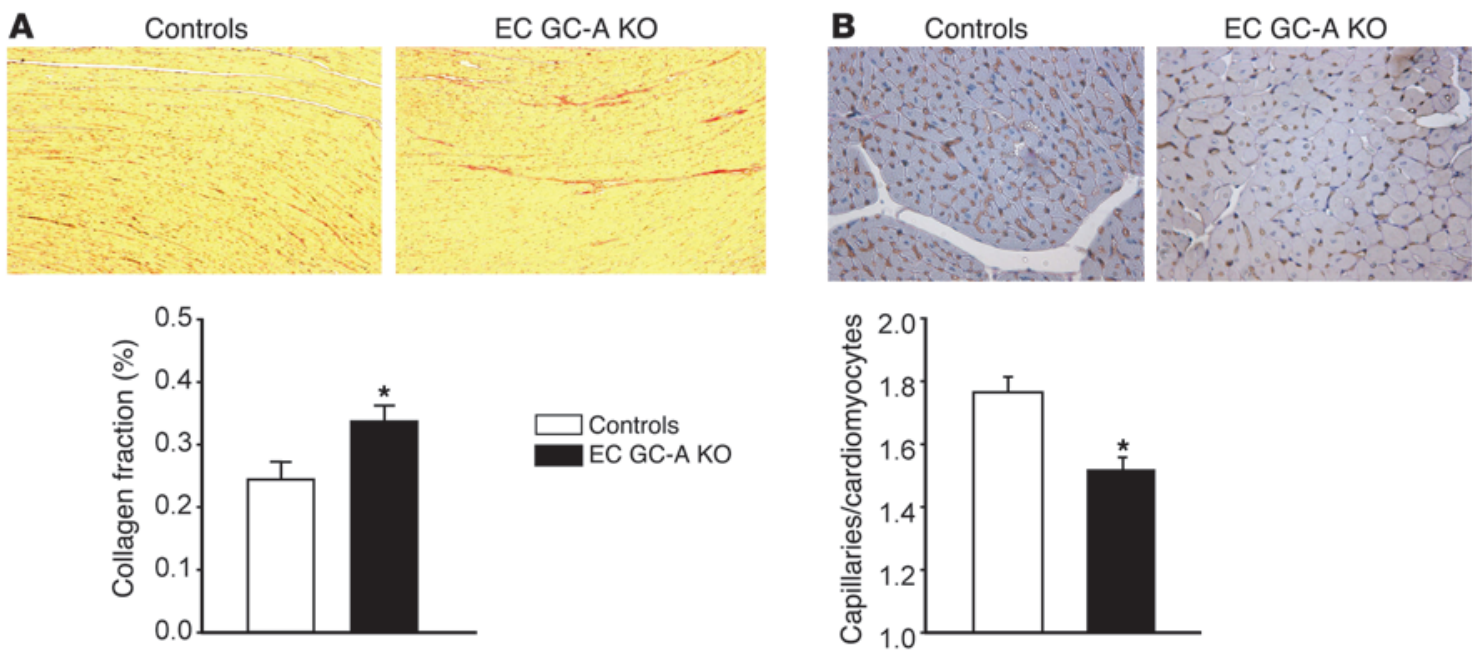

\section{Figure 5}

Cardiac vascular rarefaction and fibrosis in EC GC-A KO mice subjected to TAC. Left-ventricular interstitial collagen (A) and capillary density (B) in control and EC GC-A KO mice were assessed 10 days after TAC. Sections were stained with the endothelial marker isolectin (brown) and PAS for quantification of capillary density and myocytes and with picrosirius red for quantification of interstitial collagen fractions. Top: Representative cardiac sections demonstrating increased interstitial fibrosis and fewer capillaries in the hearts of EC GC-A KO as compared with respective control littermates. Original magnification, $\times 200$ (A) and $\times 400$ (B). Bottom: Data from 14 animals per group are summarized. In B, capillary density is expressed as the number of capillaries per myocyte. ${ }^{*} P<0.05$ versus control mice. 


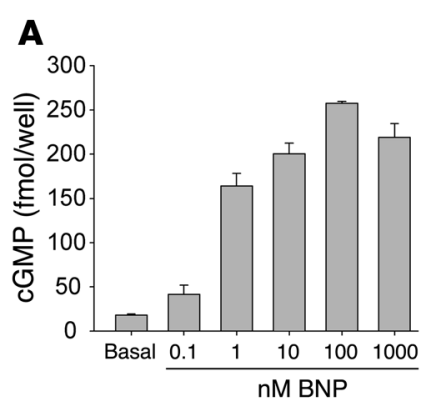

B

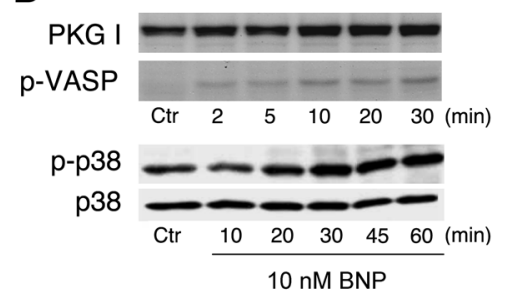

C
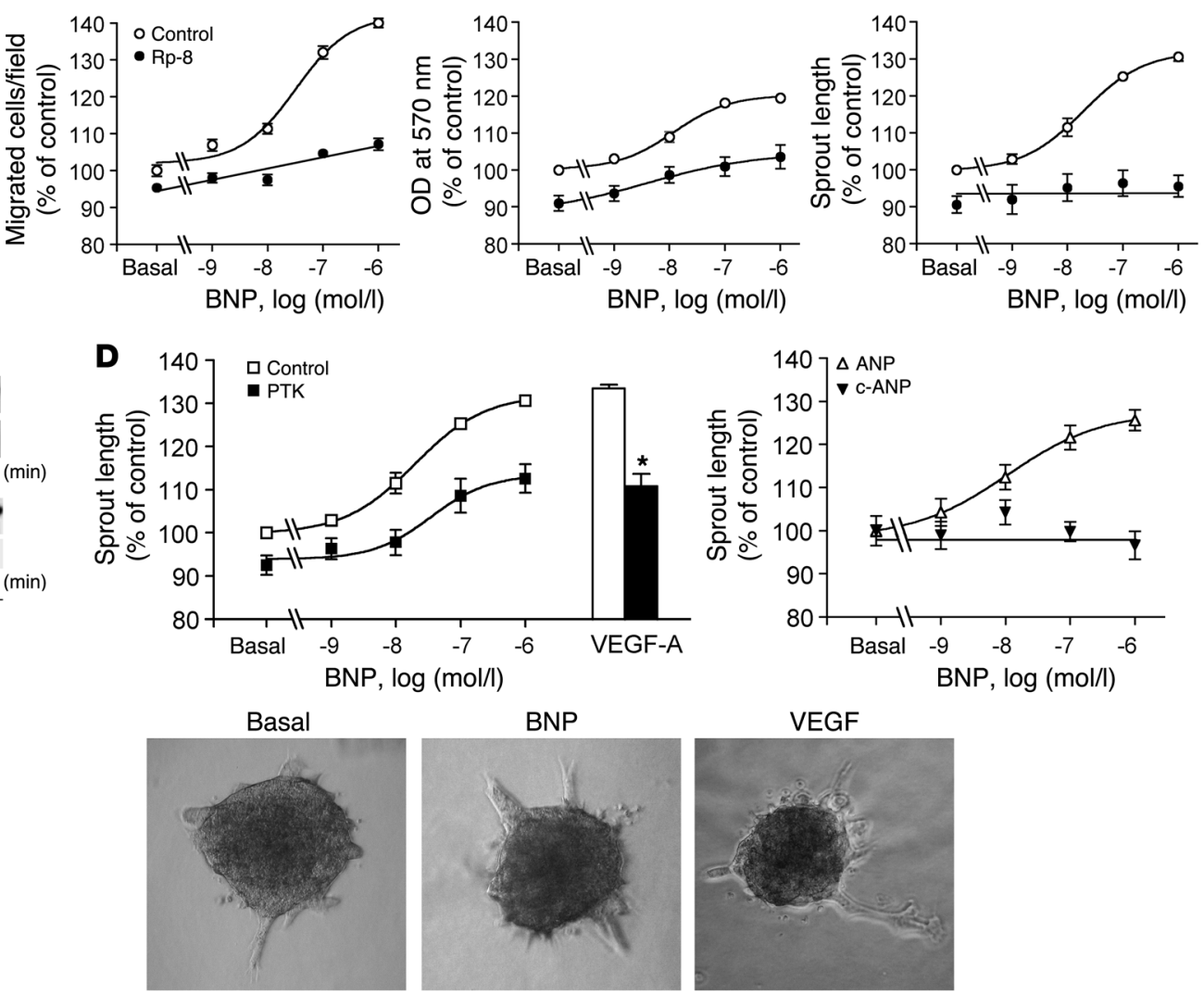

\section{Figure 6}

BNP induces angiogenic responses in cultured RFPECs. (A) BNP (0.1 nM to $1 \mu \mathrm{M}, 5$ minutes) increased intracellular cGMP. (B) Western blot analyses showed expression of PKG I and time-dependent changes in phospho-VASP and phospho-p38 in response to BNP. (C) Effects of increasing concentrations of BNP $(0.1 \mathrm{nM}$ to $1 \mu \mathrm{M})$ on (left to right): migration (4 hours), proliferation (24 hours), and sprouting (24 hours) in the absence and presence of the PKG I inhibitor Rp-8-pCPT-cGMPs (Rp-8, $10 \mu \mathrm{M})$. Basal migration, proliferation, and sprouting of untreated cells was set to $100 \%$. (D) Effects $50 \mathrm{ng} / \mathrm{ml}$ VEGF-A versus increasing concentrations of BNP, ANP, and cANP $4-23$ on the sprouting behavior of RFPECs. Where indicated, $100 \mathrm{nM}$ of the specific inhibitor of VEGF receptor tyrosine kinases PTK787/ZK222584 (PTK) (24) was present. Data are shown as the mean \pm SEM of 3 independent experiments performed using $3-10$ samples. ${ }^{*} P<0.05$ versus VEGF-A in the absence of PTK. Pictures in D: original magnification, $\times 10$.

prominent within 2 minutes of BNP treatment and raised with longer incubation times (Figure 7C, top). Last, we examined the expression and phosphorylation of the proliferative MAPK signaling cascades ERK1/2 and p38. BNP $(10 \mathrm{nM})$ provoked a mild but significant increase in the levels of phosphorylated p38 within 15 minutes (Figure 7C). This effect was abolished in GC-A-deficient MLECs (Figure 7C). No significant changes were observed in the levels of phosphorylated ERK1/2 (Figure 7C) and Akt (data not shown) in response to BNP (10 nM, incubation of cells up to 60 minutes). Thus, similar responses to BNP (stimulation of VASP and phosphorylation of p38 [albeit delayed] but not of ERK1/2) were observed in RFPECs (Figure 6B) and in MLECs.

\section{Discussion}

The endogenous $N P / G C-A$ system promotes angiogenesis. Here we identify what we believe to be a novel function for the NP/GC-A system as a regulator of angiogenesis in the heart and skeletal muscle. Our first indication that endogenous ANP and/or BNP might be proangiogenic came from studies in mice with global deletion of GC-A that showed severely delayed and impaired restoration of blood flow in hind limb ischemia. Within the vascular system, GC-A is densely expressed on both SMCs and ECs (1). We have shown in previous studies that SMC-restricted deletion of GC-A in mice (SMC GC-A KO) completely abolished the direct vasodilating effects of ANP and BNP (13), whereas endotheliumrestricted GC-A deletion (EC GC-A KO) did not affect NP-induced vasodilatation (14). Notably, postischemic hind limb reperfusion was fully preserved in SMC GC-A KO but severely diminished in EC GC-A KO mice, demonstrating that the protective effect of the NP/GC-A system in this setting is not due to a vasodilatory action. In fact, postischemic reperfusion was impaired to a similar extent in mice with global and with endothelial GC-A deletion, indicating that vascular ECs are the main cell type mediating the protective effects of NPs in this situation. Furthermore, we observed that ANP and BNP increased proliferation and migration of cultured microvascular ECs by activating PKG I. These results indicated that NPs can stimulate endothelial regeneration independent of the VEGF-A/VEGFR2 system. 
A

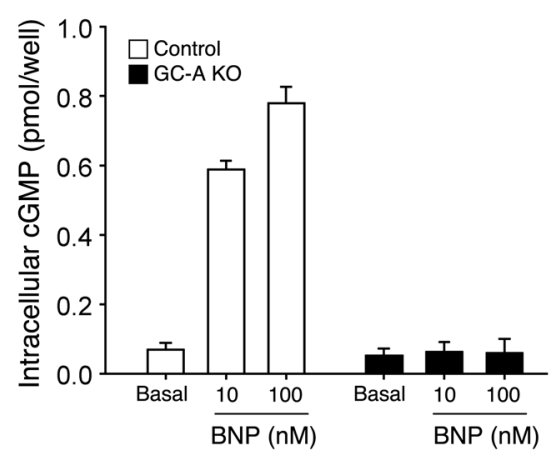

B
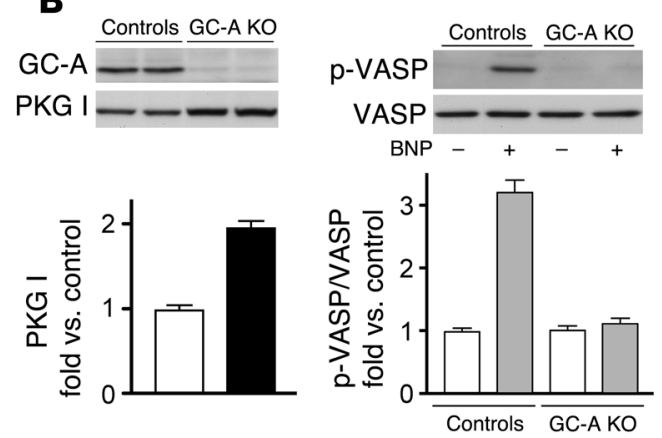

C
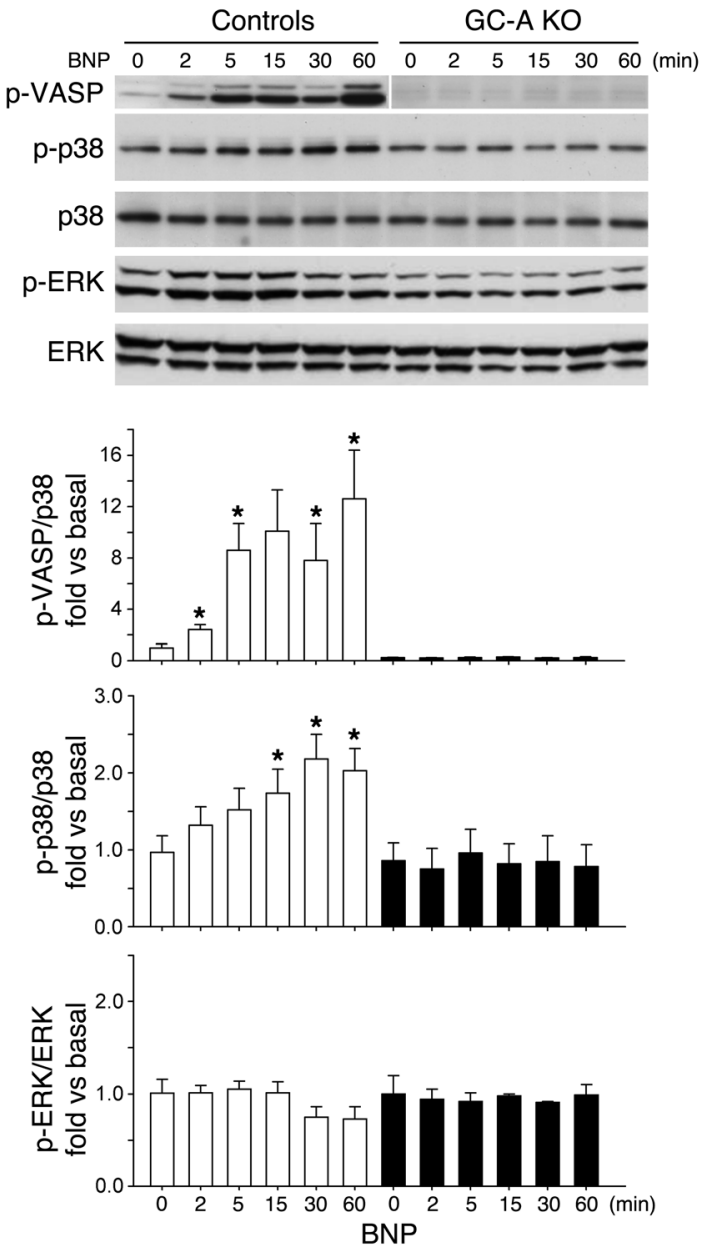

Figure 7

BNP stimulates phosphorylation of VASP and p38 MAPK in primary cultured MLECs via the GC-A receptor. (A) BNP (10 and $100 \mathrm{nM}, 5$ minutes) increased cGMP in wild-type (control) but not GC-A-deficient MLECs. (B) Western blot analyses. GC-A protein was present in wild-type and absent in GC-A-deficient ECs. PKG I was present in MLECs of both genotypes, expression levels being significantly increased in GC-A-deficient cells. BNP (10 nM, 5 minutes) stimulated the phosphorylation of VASP at the PKG I preferred site in wild-type but not in GC-A KO MLECs ( $n=3$ in all experiments). (C) Western blot analyses showing the time-dependent changes in phosphorylated VASP and p38 in response to 10 nM BNP. Top: Representative immunoblots of phospho-VASP, phospho-p38, total p38, phospho-ERK1/2, and total ERK1/2. The expression of phospho-VASP in controls and GC-A KO was evaluated in 2 separate gels as indicated by the separating white line. Bottom: Protein levels of phospho-VASP and phospho-p38 were normalized to total p38 and those of phospho-ERK1/2 to total ERK1/2 and were calculated as fold relative to untreated cells (time 0). Phospho-VASP and phospho-p38 levels were significantly increased by BNP in control but not in GC-A-deficient MLECs ( $n=3$ experiments; ${ }^{*} P<0.05$ vs. basal at 0 minutes). Phospho-ERK $1 / 2$ levels were not affected by BNP.

Blood flow recovery in this animal model of chronic hind limb ischemia depends on both angiogenesis and arteriogenesis (12). Whereas angiogenesis is driven primarily by hypoxia (25), arteriogenesis is initially impelled by hemodynamic forces and is a requisite for functional recovery $(12,25,26)$. Vascular insufficiency results primarily from large vessel disease, and efficient reperfusion requires induction of arteriogenesis in addition to angiogenesis (25). As demonstrated by histology and MRA, both processes were significantly impaired in EC GC-A KO mice. As shown, NPs induce endothelial proliferation and migration in vitro and therefore seem to be primarily involved in angiogenesis. However, the expansion of collateral vessels to form functional conduits is complex, involving proliferation and maturation of multiple cell types and vessel wall components, including SMCs and fibroblasts, which also express GC-A receptors (1). The fact that ischemic reperfusion was severely impaired in mice with global or endothelium-restricted GC-A ablation, but not in SMC GC-A KO mice, suggests that the mitogenic activity of NPs is endothelium specific. Moreover, at least in vitro, NPs have been shown to even inhibit cell growth of fibroblasts (27) and vascular SMCs (28). Therefore, induction of arteriogenesis by the NP/GC-A system in vivo might be achieved by endothelial release of factors that act upon other cell types or by an indirect action on the vascular bed, such as inducing alterations in hemodynamic forces through distal angiogenesis $(25,26)$.

Several studies involving additional cellular players focused on the participation of inflammation in angiogenesis. Monocyte activation with the production of cytokines might associate with angiogenesis in a rabbit hind limb ischemia model (29). Infarct size after myocardial ischemia/reperfusion injury was smaller in mice with global GC-A gene deletion, accompanied with decreases 
in neutrophil activation (30). In light of these results, the genotype-dependent differences in angiogenesis reported herein could result in part from potential proinflammatory effects of NPs. However, we confirmed that the number of infiltrating leukocytes in the ischemic limb of GC-A-deficient mice was similar to that in respective controls. This finding supports the notion that direct endothelial effects of NPs, and not possible proinflammatory effects, play a role in angiogenesis.

$B N P$ is produced in activated satellite cells of the ischemic hind limb muscle. Whereas ANP is mainly produced in cardiomyocytes, BNP production has been demonstrated in many other cell types. As one example, immunohistochemistry revealed that fibroblasts, macrophages, and infiltrating neutrophils are sources of BNP in the heart after myocardial infarction (31). We therefore postulated that inflammatory cells could be a source for local BNP production in ischemic hind limb. However, our immunohistochemical studies did not reveal the presence of BNP in infiltrating CD45positive inflammatory cells. In contrast, we observed that satellite cells, activated within the ischemic hind limb early after ischemia, display BNP immunoreactivity. Remarkably, quiescent satellite cells detected in the nonischemic muscle or within the ischemic muscle at later time points (2 weeks after ischemia) did not contain BNP. These findings were corroborated by RT-PCR analyses of BNP mRNA expression in laser-microdissected cells, which also showed that BNP is expressed in satellite cells within the ischemic muscle but not in quiescent cells of the nonischemic limb. The satellite cell is the principal self-renewing myogenic stem cell in the skeletal muscle (16). In the adult, satellite cells are mitotically quiescent and reside in a niche between the basal lamina and the sarcolemma of their associated muscle fibers. They become activated in response to stress such as ischemic injury and hypoxia (16). The progeny of activated satellite cells regenerates damaged skeletal muscle fibers (16). New reports demonstrated the continuous relationship of satellite cells with endothelial cells (32). Our data support the notion that BNP produced by satellite cells proliferating in the ischemic muscle may stimulate the regeneration of neighboring endothelia. Recent studies demonstrated that hypoxia, via stabilization of the hypoxia-inducible factor HIF- $1 \alpha$, induces BNP expression in different cells $(8,33)$. In line with these observations, quantitative RT-PCR analyses demonstrated that BNP mRNA expression in cultured C2C12 cells, a myoblast cell line, was stimulated by hypoxia. Taken together, our studies in vivo and in vitro suggest that satellite cells express BNP mainly when they become activated by ischemic injury and hypoxia.

Cardiac natriuretic peptides regulate coronary angiogenesis in response to pressure load bypertrophy. Cardiac hypertrophy and angiogenesis are coordinately regulated during adaptive cardiac growth, and disruption of the balanced growth and angiogenesis leads to contractile dysfunction and heart failure (17). This coordination is at least partly achieved by the secretion of angiogenic factors from myocytes in response to hypertrophic stimuli (17). GATA4 acts as a stress-responsive transcription factor in murine cardiomyocytes that induces the expression of angiogenic factors (34). ANP and especially BNP are among the very first factors induced in cardiomyocytes in response to pressure load or hypertrophic stimuli such as angiotensin II (35), and this induction is mediated by GATA4 (36). Our study shows in a pressure overload-induced hypertrophy model that selective disruption of the endothelial GC-A-mediated effects of NPs provokes mild cardiac capillary rarefaction, fibrosis, and diastolic dysfunction. This indicates that cardiac NPs are among the proangiogenic genes induced by GATA4 in the early phase of load-induced cardiac hypertrophy.

$N P$ stimulate endothelial proliferation and migration via GC-A/cGMP/ PKG I signaling. To supplement these in vivo findings, we performed several in vitro experiments. In cultured microvascular RFPECs, NPs significantly increased proliferation, migration, and sprouting in a concentration-dependent and GC-A/cGMP/PKG I-mediated manner. Notably, the extent of the maximal angiogenic effect of NPs was similar to that of VEGF-A. Furthermore, we observed that the effects of NPs are not mediated by the endothelial release of VEGF-A and autocrine/paracrine activation of the VEGF receptor. Together with published observations in macrovascular ECs (9), these results indicate that NPs can act directly on ECs and potentiate endothelial regeneration. One possible action of endothelial GC-A/cGMP/PKG I signaling is the phosphorylation of VASP, a protein associated with focal adhesion sites and adherens junctions (37). Our experiments with primary cultured MLECs obtained from wild-type and GC-A-deficient mice as well as the experiments in RFPECs showed that BNP, via GC-A/cGMP and PKG I, leads to VASP phosphorylation in microvascular endothelia. In macrovascular human umbilical vein ECs (HUVECs), this pathway has been shown to be important for reorganization of the actin cytoskeleton and endothelial tube formation (37). Other studies in HUVECs showed that PKG phosphorylates $\mathrm{p} 21$-activated kinase 1 (Pak1), thereby stimulating Pak/VASP association, which also seems to be important for endothelial migration (38). Members of the MAPK family, including ERK1/2 and p38 MAPK, and the serine/threonine kinase Akt/PKB are also important mediators of endothelial growth and migration (39). In contrast to published observations in macrovascular ECs (9), BNP did not affect the levels of phospho-Akt or phospho-ERK1/2 in microvascular MLECs and RFPECs. Instead, the levels of phospho-p38 were mildly but significantly increased in response to BNP. p38 MAPK mediates proliferation and migration in human ECs induced by mitogens such as VEGF-A (40). In line with our observations, a selective induction of phospho-p38 (and not of phospho-ERK1/2) by NPs has been observed in other tissues (41). Taken together, our data suggest that activation of VASP and of p38 MAPK cooperate in the NP/GC-A-dependent stimulation of processes required for angiogenesis, such as endothelial proliferation and migration.

In conclusion, results of the present study demonstrate that the endothelial GC-A receptor for ANP and BNP is critically involved in ischemic skeletal muscle angiogenesis and also in cardiac angiogenesis accompanying compensated pressure load-induced hypertrophy. Altered cGMP signaling is a common feature of patients with lower extremity peripheral artery disease (42) and patients with cardiac hypertrophy/heart failure (1). Our studies of EC GC-A KO mice indicated that an inhibition of the endothelial $\mathrm{NP} / \mathrm{GC}-\mathrm{A} / \mathrm{cGMP}$ effects could accelerate the progression of these diseases and support further work to assess the importance of the NP/GC-A system, particularly its endothelial alterations, in human cardiovascular diseases.

\section{Methods}

Genetic mouse models. Mice with global deletion of GC-A (GC-A KO) and corresponding wild-type mice were generated by the group of D.L. Garbers (University of Texas Southwestern Medical Center, Dallas, Texas, USA) (3). Mice with conditional endothelial (EC GC-A KO) or smooth musclerestricted deletion of GC-A (SMC GC-A KO mice) and control littermates

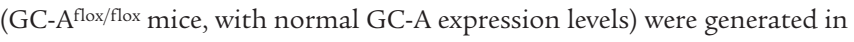


our laboratory and genotyped as described previously $(13,14)$. Male and female mice homozygous for floxed GC-A and heterozygous for the Tie2-Cre transgene (EC GC-A KO; ref. 14) or the SM22-Cre transgene (SMC GC-A KO; ref. 13) were used in our studies. Their littermates with floxed GC-A served as controls. All study mice were approximately 2 months old. The experiments were performed in accordance with German legislation on protection of animals and the Guide for the care and use of laboratory animals (NIH publication no. 85-23. Revised 1985) and were approved by the local governmental animal care committee (Regierung von Unterfranken, Würzburg).

Hind limb ischemia. Mice were anesthetized with isoflurane (2\%). The left femoral artery was exposed proximal to the origin of the arteria poplitea, dissected free, and excised. Hind limb blood flow was measured before, immediately after, and 3, 7, 14, 21, 28, and 35 days after occlusion, using the same anesthesia. The animals were placed for 15 minutes on $37^{\circ} \mathrm{C}$ heated pads. An LDPI (Moor Instruments) was used to estimate relative blood flow. Ratios of occluded to nonoccluded values were compared after background subtraction (10).

High-resolution MRA. MRA investigations were performed using a vertical Bruker DRX 9.4 Tesla wide-bore NMR spectrometer equipped with a 40-mm gradient set (capable of $1 \mathrm{~T} / \mathrm{m}$ maximum gradient strength) and a linearly driven $30-\mathrm{mm}$ birdcage resonator. Mice were anesthetized with $1.5 \%$ isoflurane and kept at $37^{\circ} \mathrm{C}$. $3 \mathrm{D}$ MRA data sets were acquired using flow-compensated gradient echo sequences without any contrast agent (15). For normalization of collateral vessel volumes, a defined area of the contralateral uninjured femoral artery between the proximal end (identical to the last branching of the iliac artery) and the branching into the popliteal artery was segmented. Data were calculated as ratios of the newly developed collateral vessels in the ischemic hind limb to this reference segment in the nonischemic hind limb (15).

Histology and immunohistochemistry. Hearts and hind limb muscles of EC GC-A KO mice and their control littermates were fixed in $4 \%$ paraformaldehyde and embedded in paraffin. Two-micrometer sections were used for stainings. ECs were stained with biotinylated GSL I-isolectin B4 (dilution 1:500; D1205; Vector Laboratories) and detected by streptavidin peroxidase (POX). The sections were counterstained with diastase PAS to visualize the cell membrane of individual skeletal or cardiomyocytes. Local expression of BNP in the ischemic adductor muscle was assessed by immunohistochemical staining. After pretreatment with citrate buffer $\left(\mathrm{pH} 6.0,30\right.$ minutes at $95^{\circ} \mathrm{C}$ ), the specific antibodies against BNP (dilution 1:1,000; T-4207; Bachem) and Myf5 (1:2,500; Santa Cruz Biotechnology Inc.) were applied and detected with donkey anti-rabbit biotin (1:500; Dianova). The antibody against mouse CD45 (1:2,500; clone 30-F11; eBioscience) was detected by mouse anti-rat biotin (1:100; Dianova). The antibody complexes were developed with streptavidin POX. The sections were counterstained with H\&E. Negative controls were performed by using the appropriate polyclonal immunoglobulin or by avoiding the primary antibody.

Morphometrical analyses of capillary density, inflammation, and cardiac fibrosis. In each animal, 5-6 fields from 6 different sections were photographed with a digital camera (CCD-FV2T; Olympus). By computer-assisted analysis with the Cell ${ }^{\mathrm{D}}$ system (Olympus), capillary density was calculated as the number of capillaries (stained with isolectin) per myocyte. The mean number of infiltrating CD45-positive leukocytes was determined within areas of muscle necrosis or regeneration, and the ratio was determined as the assessment of inflammation (10). Cardiac interstitial collagen fractions were evaluated on PAS- or picrosirius red-stained left-ventricular sections as described previously (43).

Laser microdissection and RT-PCR. Paraffin-embedded $2-\mu \mathrm{m}$ sections were placed on membrane slides (Zeiss) and immunohistochemically stained against Myf5. Using a PALM MicroBeam device (Zeiss), we captured 80-100 Myf5-immunorective satellite cells or Myf5-negative muscle fibers. Total
RNA was extracted with the RNeasy FFPE kit (QIAGEN). First-strand cDNA was synthesized using RevertAid M-MuLV Reverse Transcriptase (Fermentas Life Sciences) and random hexamers. One microliter of the cDNA pool was subjected to PCR with specific primers for BNP (sense, 5'ATCTGTCACCGCTGGGAGGTC; antisense, 5'-TCTTATCAGCTCCAGCAGCTT; 92-bp product), with mouse cyclophilin used as reference gene.

RT-PCR analyses in C2C12 cells. C2C12 cells, a mouse myoblast cell line (ATCC), were cultivated to $80 \%$ confluence in $35-\mathrm{mm}$ dishes. The medium was buffered with $20 \mathrm{mM}$ HEPES to adjust to a $\mathrm{pH}$ of 7.4. In hypoxia experiments, cells were gassed with $95 \% \mathrm{~N}_{2}$ and $5 \% \mathrm{CO}_{2}$ for up to 8 hours. Total RNA was extracted with TRIzol reagent. First-strand cDNA was synthesized from $1 \mu \mathrm{g}$ total RNA using SuperScript II RNase $\mathrm{H}^{-}$Reverse Transcriptase (Invitrogen) and random primers. SYBR green I-based quantitative real-time RT-PCR for BNP was performed (sense primer, 5'-AAGCTGCTGGAGCTGATAAGA; antisense primer, 5'-GTTACAGCCAAACGACTGAC; 222-bp product), with mouse cyclophilin used as reference gene.

Echocardiography and TAC. Mice were anesthetized with a combination of ketamine $(50 \mathrm{mg} / \mathrm{kg}$ body weight, intraperitoneally) and isoflurane (2.5\%), and pressure overload was surgically induced by TAC as previously described (43). Five days before and 10 days after TAC, animals were anesthetized with $2 \%$ isoflurane. Doppler echocardiography was performed with a digital cardiac ultrasound machine equipped with a $15-\mathrm{MHz}$ linear phased-array transducer and a 12-MHz short-focal-length phased array transducer (SONOS 5500, C1 software package; Philips Healthcare) (44).

Culture of primary $M L E C$. ECs from peripheral lung tissue from wild-type $\left(G C-A^{+/+}\right)$and $G C-A$-deficient mice were isolated and cultured as described previously (45). After 3-4 days, cells were trypsinized and endothelial cells were selected with magnetic beads coated with anti-mouse CD102 antibody (BD). Cultures were grown to confluence and selected a second time before being plated for experiments (45). Immunocytochemistry with antibodies against 3 different endothelial markers (VE-cadherin, von Willebrand factor, and claudin-5) demonstrated that after the second selection, more than $95 \%$ of cultured cells were endothelial.

Culture of microvascular RFPECs. RFPECs (a gift from A. Horowitz, Dartmouth Medical School, Lebanon, New Hampshire, USA; ref. 19) were cultured in DMEM containing 10\% FCS (19). All experiments were conducted in serum-reduced DMEM (0.5\% FCS).

Determination of endothelial cGMP contents. ECs were incubated with synthetic BNP (American Peptide Company Inc.) for 5 minutes. Then the incubation medium was aspirated, and either intracellular cGMP was extracted with icecold $70 \%$ (v/v) ethanol or the cells were lysed for protein extraction (see below). Cellular cGMP contents were quantified by radioimmunoassay (45).

Western blotting. Endothelial proteins were resolved by $10 \%$ SDS-PAGE. Electrophoresis and immunoblotting were performed as described previously (45). The primary antibodies were against GC-A and PKG I (generated in our laboratories; ref. 45), the cytoskeletal protein VASP, VASP phosphorylated at the PKG I-preferred site (mouse Ser235, rat Ser236; ref. 20), and phosphorylated/ unphosphorylated MAPKs (all Cell Signaling Technology).

Proliferation and migration assays. The incorporation of 3-(4,5-dimethylthiazol-2-yl)-2,5-diphenyltetrazolium bromide (MTT; Sigma-Aldrich) was used to asses cell proliferation. Briefly, 5,000 cells per well were seeded in a 96-well plate and cultured for 48 hours before incubation with or without NPs for an additional 24 hours. MTT $(5 \mathrm{mg} / \mathrm{ml})$ was added, and medium was removed 4 hours later. The colored formazan product was resolved in $\mathrm{HCl} 0.04 \mathrm{~N} /$ isopropanol and quantified by absorbance measurements at $570 \mathrm{~nm}$. Transwell cell migration assays were performed using a 96-well Boyden chemotaxis apparatus (Neuro Probe) (46). Briefly, polycarbonate filters with $8-\mu \mathrm{m}$ pore size (Neuro Probe) were coated with $100 \mu \mathrm{g} / \mathrm{ml}$ type I collagen overnight. RFPECs (5,000/well) were incubated with test agents for 4 hours, and thereafter, the filter was fixed with 
methanol and stained with Giemsa. RFPECs were also used to perform an in vitro angiogenesis sprouting assay (47). Spheroids generated for 24 hours with 400 cells were embedded into a 3D collagen-based gel placed in a 48-well plate. They were stimulated under different conditions for 24 hours, and the cumulative sprout length was measured for each condition. These experiments were performed in the absence and presence of PTK787/ZK222584, an inhibitor of VEGF receptor tyrosine kinases (24), which was kindly provided by Novartis.

VEGF secretion. RFPECs (50,000/well) were seeded in a 24-well plate and cultured for 48 hours before incubation with/without NPs for an additional 4 or 24 hours. Then the supernatants were collected, centrifuged, and stored at $-20^{\circ} \mathrm{C}$. VEGF-A concentrations were determined by immunoassay (Quantikine; R\&D Systems).

Statistics. Results are presented as mean \pm SEM. Group data were compared by ANOVA (with genotype and treatment as categories) followed by the multiple-comparison Bonferroni $t$ test to assess differences between groups. $P$ values less than 0.05 were considered statistically significant.

\section{Acknowledgments}

This study was supported by Deutsche Forschungsgemeinschaft, Sonderforschungsbereiche 688 (to M. Kuhn and S. Gambaryan), 612 (to U. Flögel and J. Schrader), TR23 (to T. Wieland), BA 1730/9-1 (to H.A. Baba), and 656 (to J. Stypmann) and by the Interdisziplinäres Zentrum für Klinische Forschung Münster (to J. Stypmann). The authors thank Birgit Gassner, Alexandra Gazinski, Dorothe Möllmann, and Mareike Müller for excellent technical support.

Received for publication September 11, 2008, and accepted in revised form April 8, 2009.

Address correspondence to: Michaela Kuhn, Physiologisches Institut der Universität Würzburg, Röntgenring 9, D-97070 Würzburg, Germany. Phone: 49-931-31-2721; Fax: 49-931-31-2741; E-mail: michaela.kuhn@mail.uni-wuerzburg.de.
1. Kuhn, M. 2003. Structure, regulation, and function of mammalian membrane guanylyl cyclase receptors, with a focus on guanylyl cyclase-A. Circ. Res. 93:700-709.

2. John, S.W., et al. 1995. Genetic decreases in atrial natriuretic peptide and salt-sensitive hypertension. Science. 267:679-681.

3. Lopez, M.J., et al. 1995. Salt-resistant hypertension in mice lacking the guanylyl cyclase-A receptor for atrial natriuretic peptide. Nature. 378:65-68.

4. Skryabin, B.V., et al. 2004. Hypervolemic hypertension in mice with systemic inactivation of the (floxed) guanylyl cyclase-A gene by alphaMHC-Cremediated recombination. Genesis. 39:288-298.

5. Tamura, N., et al. 2000. Cardiac fibrosis in mice lacking brain natriuretic peptide. Proc. Natl. Acad. Sci. U. S. A. 97:4239-4244.

6. Bennett, B.D., et al. 1991. Extracellular domainIgG fusion proteins for three human natriuretic peptide receptors. J. Biol. Chem. 266:23060-23067.

7. De Bold, A.J., et al. 2001. The physiological and pathophysiological modulation of the endocrine function of the heart. Can. J. Physiol. Pharmacol. 79:705-714.

8. Weidemann, A., et al. 2008. Hypoxia, via stabilization of the hypoxia-inducible factor HIF-1alpha, is a direct and sufficient stimulus for brain-type natriuretic peptide induction. Biochem. J. 409:233-242.

9. Kook, H., et al. 2003. Physiological concentration of atrial natriuretic peptide induces endothelial regeneration in vitro. Am. J. Physiol. Heart Circ. Physiol. 284:H1388-H1397.

10. Yamahara, K., et al. 2003. Significance and therapeutic potential of the natriuretic peptides/cGMP/ cGMP-dependent protein kinase pathway in vascular regeneration. Proc. Natl. Acad. Sci. U. S. A. 100:3404-3409.

11. Park, K., et al. 2008. Therapeutic potential of atrial natriuretic peptide administration on peripheral arterial diseases. Endocrinology. 149:483-491.

12. Scholz, D., et al. 2002. Contribution of arteriogenesis and angiogenesis to postocclusive hindlimb perfusion in mice. J. Mol. Cell. Cardiol. 34:775-787.

13. Holtwick, R., et al. 2002. Smooth muscle-selective deletion of guanylyl cyclase-A prevents the acute but not chronic effects of ANP on blood pressure. Proc. Natl. Acad. Sci. U. S. A. 99:7142-7147.

14. Sabrane, K., et al. 2005. Vascular endothelium is critically involved in the hypotensive and hypovolemic actions of atrial natriuretic peptide. J. Clin. Invest. 115:1666-1674.

15. Jacoby, C., et al. 2008. Dynamic changes in murine vessel geometry assessed by high resolution magnetic resonance angiography: a $9.4 \mathrm{~T}$ study. J. Magn. Reson. Imaging. 28:637-645.
16. Le Grand, F., and Rudnicki, M.A. 2007. Skeletal muscle satellite cells and adult myogenesis. Curr. Opin. Cell Biol. 19:628-633.

17. Walsh, K., and Shiojima, I. 2007. Cardiac growth and angiogenesis coordinated by intertissue interactions. J. Clin. Invest. 117:3176-3179.

18. Stypmann, J., et al. 2006. Age and gender related reference values for transthoracic Doppler-echocardiography in the anesthetized CD1 mouse. Int. J. Cardiovasc. Imaging. 22:353-362.

19. Liu, M., and Horowitz, A. 2006. A PDZ-binding motif as a critical determinant of Rho guanine exchange factor function and cell phenotype. $\mathrm{Mol}$. Biol. Cell. 17:1880-1887.

20. Smolenski, A., Poller, W., Walter, U., and Lohmann, S.M. 2000. Regulation of human endothelial cell focal adhesion sites and migration by cGMP-dependent protein kinase I. J. Biol. Chem. 275:25723-25732.

21. Pedram, A., Hu, R.M., and Levin, E.R. 1997. Vasoactive peptides modulate vascular endothelial cell growth factor production and endothelial cell proliferation and invasion. J. Biol. Chem. 272:17097-17103.

22. Ziche, M., et al. 1997. Nitric oxide synthase lies downstream from vascular endothelial growth factor-induced but not basic fibroblast growth factorinduced angiogenesis. J. Clin. Invest. 99:2625-2634.

23. Hood, J., and Granger, H.J. 1998. Protein kinase $G$ mediates vascular endothelial growth factorinduced Raf- 1 activation and proliferation in human endothelial cells. J. Biol. Chem. 273:23504-23508.

24. Wood, J.M., et al. 2000. PTK787/ZK 222584, a novel and potent inhibitor of vascular endothelial growth factor receptor tyrosine kinases, impairs vascular endothelial growth factor-induced responses and tumor growth after oral administration. Cancer Res. 60:2178-2189.

25. Van Royen, N., et al. 2001. Stimulation of arteriogenesis: a new concept for the treatment of arterial occlusive disease. Cardiovasc. Res. 49:543-553.

26. Heil, M., and Schaper, W. 2004. Influence of mechanical, cellular and molecular factors on collateral artery growth (arteriogenesis). Circ. Res. 95:449-458.

27. Cao, L., and Gardner, D.G. 1995. Natriuretic peptides inhibit DNA synthesis in cardiac fibroblasts. Hypertension. 25:227-234.

28. Ikeda, M., et al. 1997. Natriuretic peptide family as a novel antimigration factor of vascular smooth muscle cells. Arterioscler. Thromb. Vasc. Biol. 17:731-736.

29. Arras, M., et al. 1998. Monocyte activation in angiogenesis and collateral growth in the rabbit hindlimb. J. Clin. Invest. 101:40-50.

30. Izumi, T., et al. 2001. Blockade of the natriuretic peptide receptor guanylyl cyclase-A inhibits NF- $\kappa B$ activation and alleviates myocardial ischemia/reperfusion injury. J. Clin. Invest. 108:203-213.

31. Bruggink, A.H., et al. 2006. Brain natriuretic peptide is produced both by cardiomyocytes and cells infiltrating the heart in patients with severe heart failure supported by a left ventricular assist device. J. Heart Lung Transplant. 25:174-180.

32. Christov, C., et al. 2007. Muscle satellite cells and endothelial cells: close neighbors and privileged partners. Mol. Biol. Cell. 18:1397-1409.

33. Wilhide, M.E., and Jones, W.K. 2006. Potential therapeutic gene for the treatment of ischemic disease: Ad2/hypoxia-inducible factor-1alpha (HIF-1)/ VP16 enhances B-type natriuretic peptide gene expression via a HIF-1-responsive element. Mol. Pharmacol. 69:1773-1778.

34. Heineke, J., et al. 2007. Cardiomyocyte GATA4 functions as a stress-responsive regulator of angiogenesis in the murine heart. J. Clin. Invest. 117:3198-3210.

35. Majalahti, T., et al. 2007. Cardiac BNP gene activation by angiotensin II in vivo. Mol. Cell. Endocrinol. 273:59-67.

36. Marttila, M., et al. 2001. GATA4 mediates activation of the B-type natriuretic peptide gene expression in response to hemodynamic stress. Endocrinology. 142:4693-4700.

37. Chen, H., Levine, Y.C., Golan, D.E., Michel, T., and Lin, A.J. 2008. Atrial natriuretic peptide-initiated cGMP pathways regulate vasodilator-stimulated phosphoprotein phosphorylation and angiogenesis in vascular endothelium. J. Biol. Chem. 283:4439-4447.

38. Fryer, B.H., et al. 2006. cGMP-dependent protein kinase phosphorylates p21-activated kinase (Pak) 1, inhibiting Pak/Nck binding and stimulating Pak/ Vasodilator-stimulated phosphoprotein association. J. Biol. Chem. 281:11487-11495.

39. Robinson, M.J., and Cobb, M.H. 1997. Mitogenactivated protein kinase pathways. Curr. Opin. Cell Biol. 9:180-186.

40. Rousseau, S., et al. 2000. Vascular endothelial growth factor (VEGF) - driven actin-based motility is mediated by VEGFR2 and requires concerted activation of stress-activated protein kinase 2 (SAPK2/p38) and geldanamycin-sensitive phosphorylation of focal adhesion kinase. J. Biol. Chem. 275:10661-10672.

41. Keller, M., et al. 2005. Hepatocyte cytoskeleton during ischemia and reperfusion-influence of ANP-mediated p38 MAPK activation. World J. Gastroenterol. 11:7418-7429.

42. Boger, R.H., et al. 1997. Biochemical evidence for impaired nitric oxide synthesis in patients with peripheral arterial occlusive disease. Circulation. 95:2068-2074. 
43. Holtwick, R., et al. 2003. Pressure-independent cardiac hypertrophy in mice with cardiomyocyterestricted inactivation of the atrial natriuretic peptide receptor guanylyl cyclase-A. J. Clin. Invest. 111:1399-1407.

44. Stypmann, J. 2007. Doppler Ultrasound in mice. Echocardiography. 24:97-112
45. Schreier, B., et al. 2008. The heart communicates with the endothelium through the guanylyl cyclaseA receptor: acute handling of intravascular volume in response to volume expansion. Endocrinology. 149:4193-4199.

46. Benndorf, R., Böger, R.H., Ergün, S., Steenpass, A., and Wieland, T. 2003. Angiotensin II type 2 recep- tor inhibits vascular endothelial growth factorinduced migration and in vitro tube formation of human endothelial cells. Circ. Res. 93:438-447.

47. Korff, T., and Augustin, H.G. 1998. Integration of endothelial cells in multicellular spheroids prevents apoptosis and induces differentiation. J. Cell Biol. 143:1341-1352. 